\title{
ICT Environment for Multi-disciplinary Design and Multi-objective Optimisation: A Case Study
}

\author{
W.J. Vankan, R. Maas and M. ten Dam \\ National Aerospace Laboratory - NLR \\ ICT Division \\ Anthony Fokkerweg 2 \\ $1059 \mathrm{CM}$ \\ Amsterdam \\ The Netherlands \\ vankandnlr.nl
}

\begin{abstract}
This paper presents an ICT environment for multi-disciplinary design and multi objective optimisation in which a set of software tools are available for evaluation and approximation of objective functions and for proper control of several optimisation algorithms for multi objective optimisation. The ICT environment provides easy access to the relevant resources in the computer network via a Java based user interface, which can be executed stand-alone or as a Java applet based web client. As an example of application of this environment, some results of a multi objective optimisation study of a blendedwing-body aircraft configuration are shown.
\end{abstract}

\section{Introduction}

Technical product design is hardly ever an exclusively mono-disciplinary exercise. It mostly requires analyses of several different aspects of the product that are often related to different research areas or technical disciplines. For example in aeronautics, the design of an aircraft is determined by many different aspects, of which some important ones are aerodynamic behaviour, structural mechanical properties and flight mechanical and control properties [1]. For each of these aspects there is a specific technical discipline that can deal with highly detailed investigation and analysis of the design in its specific area. Such investigation is usually performed by monodisciplinary specialists who make use of a variety of dedicated analysis tools. In multi-disciplinary design it is required that these mono-disciplinary investigations effectively contribute to the integrated multi-disciplinary design process [12].

In the EU project MOB [13] the multi-disciplinary design and optimisation of a blended-wing-body (BWB) aircraft configuration is considered. For this purpose an engineering environment for multi-disciplinary design analysis is developed in MOB. This so-called computational design engine (CDE) shall be a flexible system, providing easy access to the partners in the $\mathrm{MOB}$ project and allowing them to integrate and interconnect their design and analysis tools. The CDE facilitates the multi-disciplinary design process for the BWB by transparent presentation of the necessary analysis tools, data and documents to the CDE users. The CDE also offers 
certain facilities for optimisation of the BWB design, based on an efficient but traditional approach.

When considering product design as an optimisation process where the design objectives are optimised subject to certain design constraints, multi-disciplinary design leads to mutually competing objectives and constraints that arise from the different disciplines and must be dealt with simultaneously. Such multi objective optimisation essentially differs from traditional single-objective optimisation. In this case, global or local optima do not exist for all design objectives simultaneously and hence a possibly large set of compromised optimal designs, the so-called Pareto front [8] is searched for. This requires specific search techniques, which can be considered as generalisations of single objective optimisation methods [4].

An ICT (Information and Communication Technology) environment for multidisciplinary design and multi objective optimisation has been developed in which a set of software tools are available for proper definition and approximation of objective functions and for easy control of several optimisation algorithms for multi objective optimisation. This paper presents an application of this environment to a multi objective design case of a BWB aircraft. The underlying middleware technology that is used to create this ICT environment is briefly addressed. The same underlying middleware is used in the MOB CDE, which enables easy exchange of components between these two environments. The set up of this study, in particular the methods for approximation and multi objective optimisation that have been used, will be briefly described, and some results of the design optimisation are shown.

\section{ICT environment for MDO}

Multi-disciplinary design and optimisation (MDO) requires close co-operation of a number of different technical disciplines, in each of which a variety of software tools is available for design and analysis simulations that run on specific computer platforms. The variety of software tools and the associated heterogeneous computer infrastructure and data formats impose certain complexity to close multi-disciplinary co-operation from which the end user in the MDO process should be shielded as much as possible.

Present technologies like CORBA (Common Object Request Broker Architecture), Java and WWW provide possibilities to build end-user oriented, integrated multidisciplinary design environments. These technologies can be incorporated into middleware systems in order to facilitate interoperability in MDO environments, which are usually operated on distributed heterogeneous computer networks.

SPINEware is such a middleware system that supports the construction and usage of so-called working environments on top of heterogeneous computer networks [2]. A SPINEware-based working environment can be tailored to particular end usage and application areas. A SPINEware working environment provides uniform and networktransparent access to the information, applications, and other resources available from the computer network presented to the user through an intuitive GUI: the SPINEware User Shell. SPINEware also supports web-based access to SPINEware object services via a Java applet implementation of the SPINEware User Shell [10]. 
In the MOB project SPINEware is being used for the development of the CDE, which is applied to the detailed design and optimisation of the BWB aircraft configuration. This CDE is set up as a distributed environment that is developed from contributions of each of the MOB partners. The main contributions of NLR to the CDE are in the areas of high fidelity aerodynamics, structural mechanics and flight mechanics. Other areas are contributed by other partners. For example, CAD and geometry generation are provided by the University of Delft and low fidelity aerodynamics by the University of Cranfield. Currently, the CDE components from NLR and the universities of Delft and Cranfield are operational and can be used in an integrated multi-disciplinary design process of the BWB (Fig. 1).
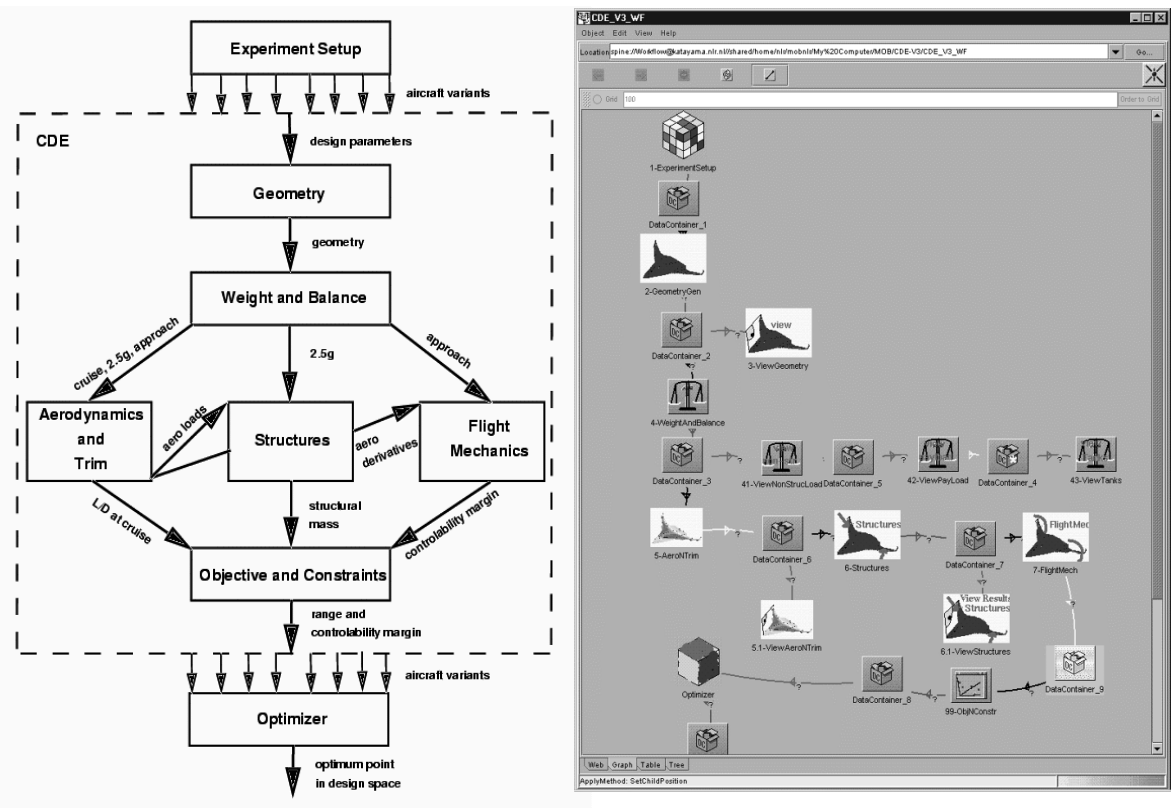

Fig. 1. Schema of the multi-disciplinary BWB design process with the currently operational MOB CDE (left) and its implementation in a SPINEware workflow (right).

At NLR the SPINEware middleware system is also being used to build user-oriented collaborative ICT environments, each dedicated to a certain application area. One such environment has been developed for the field of MDO. A set of software tools required for MDO, multi objective optimisation and approximate modeling have been integrated into this environment, and can be accessed from and executed on different computers of the heterogeneous NLR computer network. The environment is accessible via a standard web browser and comprises facilities for easy tool integration, tool manipulation and tool chaining, job and queue management, and remote operations where CORBA is used for the communication over the network. With this environment a multi objective optimisation study of the MOB BWB aircraft has been performed, where some of the analysis tools of the MOB CDE have been used (Fig. 2). 


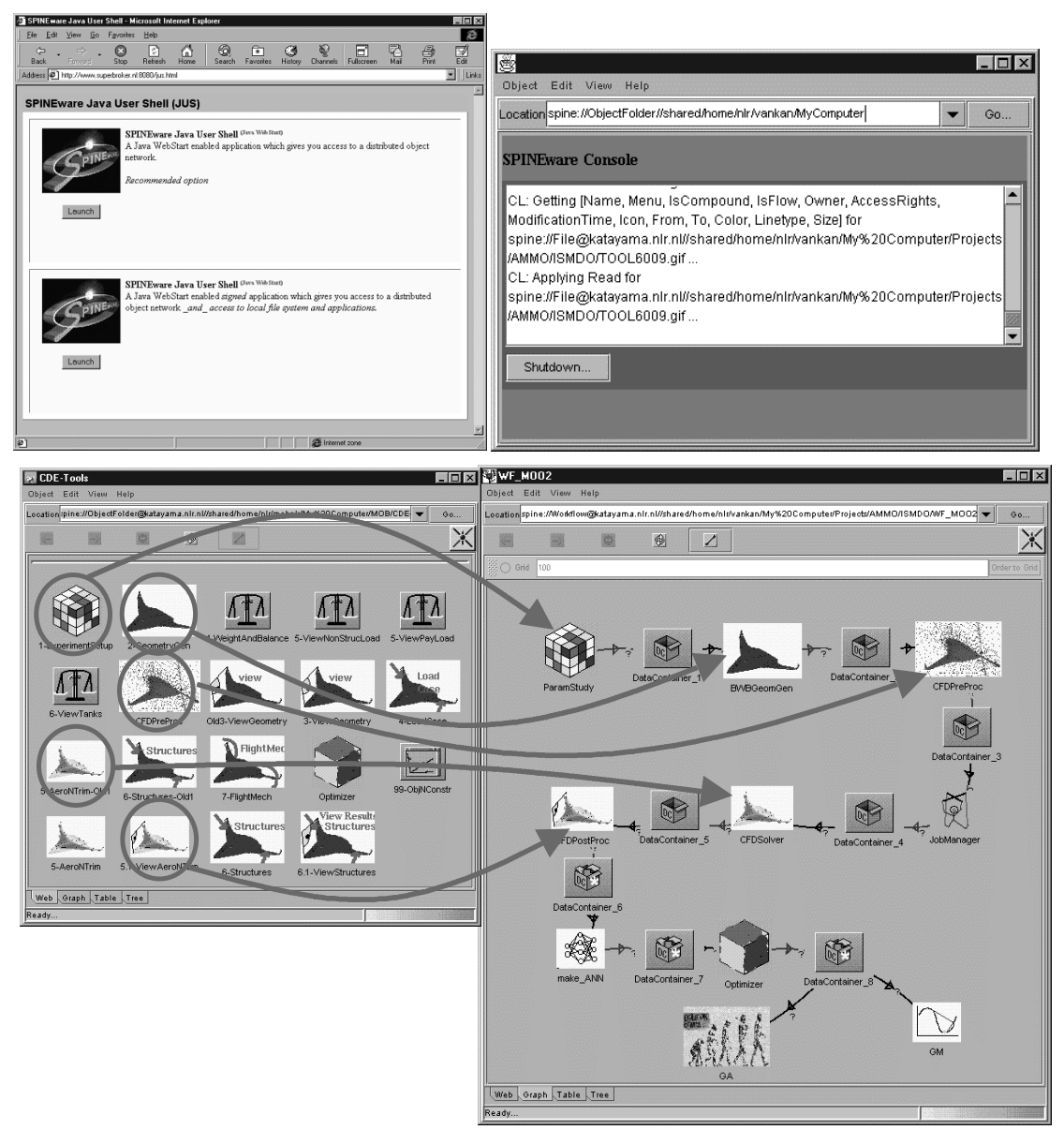

Fig. 2. Screen shots of the SPINEware working environment for MDO as accessed via a web server and using Microsoft Internet Explorer 4.0 as web client. The upper left panel shows the login web page, the upper right shows the SPINEware Console window. In the lower left panel a tool library of the MOB CDE is shown, and the lower right shows the workflow (or tool chain) for the multi objective optimisation of the BWB MDO aircraft, in which some of the $\mathrm{CDE}$ tools have been incorporated.

\section{Multi objective optimisation algorithms}

In the past decades a strong development of many different optimisation algorithms has taken place. A variety of optimisation methods, ranging from traditional gradient based optimisation methods (GM) to genetic- or evolutionary algorithms (GA), are currently widely available. In general, most GM are typically designed for single objective optimisation, while GA are more suitable for multi objective optimisation. Nevertheless it is desirable to be able to apply both methods to multi objective optimisation problems, because both have certain specific advantages. In general, the 
advantage of GA is that such algorithms have good global search capabilities, while GM easily get stuck in a local optimum. GM, on the other hand, are generally more efficient than GA in terms of the number of objective functions evaluations that is required for finding an optimum.

The application of both methods to multi objective optimisation problems is not straight-forward, and proper definition of the objective functions and effective control of the optimisation algorithm are required. In aeronautic multi-disciplinary design the evaluation of the objective functions can be computationally very expensive, for example in the case of CFD simulation of the aerodynamic behaviour. Hence a computationally much cheaper approximation model is required for such objective functions because of the large number of objective functions evaluations that is needed in multi objective optimisation [14]. Moreover, in the case of GM the multi objective optimisation process requires flexibly alternating combinations of objective and constraint functions, which can be achieved by specific control of the optimisation algorithms.

The considered MDO environment contains some specific tools for approximate modeling and multi objective optimisation. The approximate modeling tool makes use of artificial neural networks (ANN) as available from the Matlab Neural Network Toolbox [7]. Upon execution, the tool presents a user interface that guides the user through the process of setting up the ANN approximate model (Fig. 3). For multi objective optimisation, a GA and four algorithms based on GM, as available from the Matlab Optimisation Toolbox [6], can be used. The GA tool [11] applies a more or less standard MOGA technique [5], including elitism strategy and options such as niching (on both input and objective) and mating. The multi objective optimisation tool provides an interface to the four different GM based optimisation algorithms (Fig. 3).

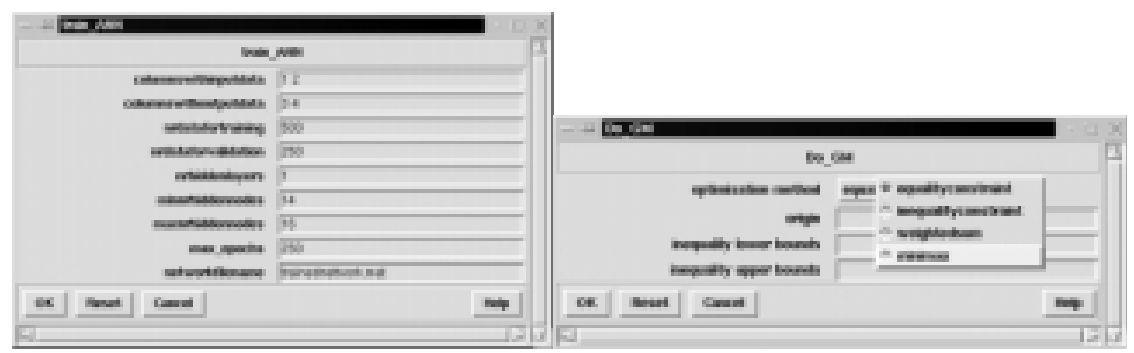

Fig. 3. The interfaces for the ANN and multi objective optimisation tools. Left: the option menu for the training of the ANN. Right: the option menu for specification and control of the GM based multi objective optimisation.

Two out of the four gradient-based methods are presented in this study. The first method is the minimax algorithm, more specific its Matlab implementation fminimax. A special strategy is applied by the multi objective optimisation tool in order to obtain the Pareto front instead of the standard single point minimax optimum. In this strategy it is assumed that each objective is positive. Furthermore it is assumed that an initial set of feasible design parameters with their corresponding objective values is 
available. The Pareto rank-one subset of this initial set is then used as demand values; this rank-one subset is the set of compromised optimal designs, also called the set of non-dominated solutions. The minimax optimisation process will then result, upon convergence of the method, in a point $P$ on the Pareto front [9] (eq. (1), Fig. 4). In formula it reads:

$$
P_{j}=c\left(p_{j}^{o p t}\right) \text { with } p_{j}^{o p t}=\underset{p \in S}{\arg \min } \max _{i} \frac{c^{i}(p)}{c^{i}\left(q_{j}\right)}
$$

where $P$ is the point on the Pareto front, $c$ is the multi-dimensional objective function, $i$ denotes $\mathrm{i}$-th the component of this objective function, $S$ is the design parameter space, $q_{j}$ is a design parameter vector corresponding to a rank-one design. If this is repeated for every rank-one point in the design parameter space, a set of points on the Pareto front is obtained.

In the second gradient based method, equality-constraint, the rank-one points of the initial set of feasible design parameters are again the starting point, but here only one objective is minimised while keeping the other fixed (Fig. 4). The implementation that has been chosen is a constrained minimisation using the Matlab function fmincon.
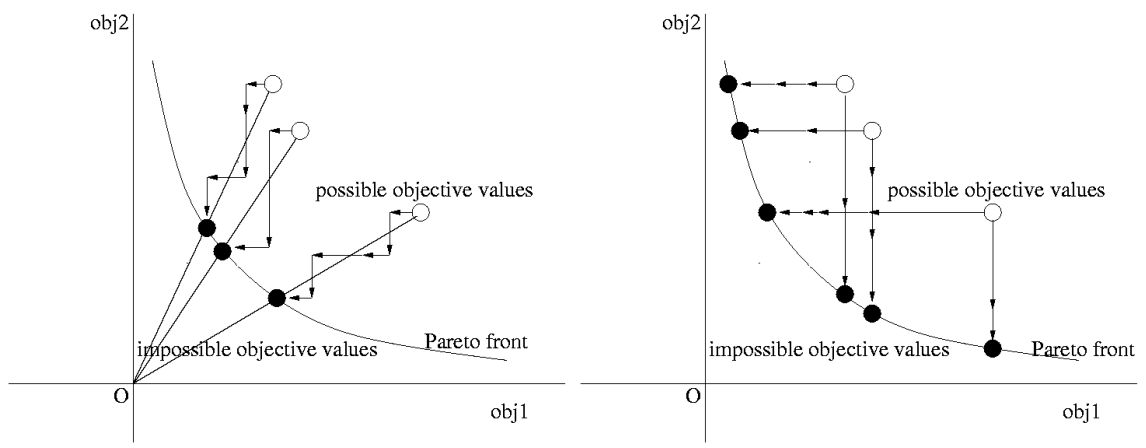

Fig. 4. Illustration of the two GM based multi objective optimisation processes. Left: using the minimax algorithm; right: using the constrained minimisation algorithm. The open circles are the initial objective values and the closed circles the final values. The arrows denote the optimisation processes.

\section{MDO of a blended-wing-body aircraft}

In the MOB project a detailed MDO study is conducted on a new BWB aircraft configuration. As a preliminary design analysis, a multi objective optimisation study is applied to some key properties of the BWB in cruise flight: aerodynamic performance $\left(\mathrm{C}_{\mathrm{dl}}\right)$ based on the lift and drag coefficients $\left(\mathrm{C}_{1}\right.$ and $\left.\mathrm{C}_{\mathrm{d}}\right)$, structural mechanical wing loading $\left(\mathrm{M}_{\mathrm{t}}\right)$ based on the roll and yaw moments at the centre of mass $\left(M_{r}\right.$ and $\left.M_{t}\right)$, and flight mechanical unbalance $\left(M_{p A}\right)$ based on the pitching moment at the centre of mass $\left(\mathrm{M}_{\mathrm{p}}\right)$. These properties are represented by the following three objective functions that are minimized: 


$$
\begin{aligned}
& C d l=\frac{C d}{C l} \\
& M t=\sqrt{M r^{2}+M y^{2}} \\
& M p A=|M p|
\end{aligned}
$$

The BWB design parameters that are varied relative to the BWB reference configuration, are the wing twist, wing sweep, and angle of attack in cruise flight (Fig 5).

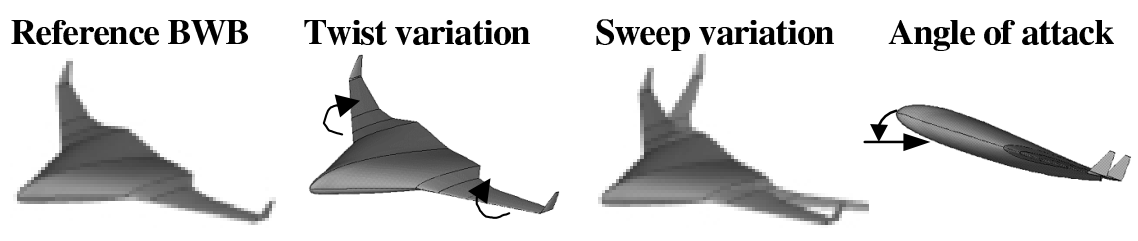

Fig. 5. Illustration of the BWB reference configuration, and the design parameters twist, sweep, and angle alpha used in the BWB pre-design study.

The objective function values can be evaluated from simulations of the airflow around the BWB under the cruise flight boundary conditions using a CFD solver for NavierStokes equations. However, because these simulations involve large scale and time consuming calculations for CAD geometry re-generation, flow domain discretisation and CFD computation, the ANN tool is used for approximation of the considered BWB properties $\left(\mathrm{C}_{1}, \mathrm{C}_{\mathrm{d}}, \mathrm{M}_{\mathrm{r}}, \mathrm{M}_{\mathrm{y}}\right.$ and $\left.\mathrm{M}_{\mathrm{p}}\right)$ from which the objectives $\mathrm{C}_{\mathrm{d}}, \mathrm{M}_{\mathrm{t}}$ and $\mathrm{M}_{\mathrm{pA}}$ are derived. For this purpose a data set of these properties is generated for the relevant ranges of the design parameters by flow simulations in a limited number of design points. The ANN has been trained using the resulting data set of 342 design points, where the three design parameters are used as ANN inputs and the five BWB properties as ANN outputs. Afterwards the outputs are combined to yield the objectives $\mathrm{C}_{\mathrm{d}}, \mathrm{M}_{\mathrm{t}}$ and $\mathrm{M}_{\mathrm{pA} .}$. To train the network the input set is split in separate training, validation and test sets of 250,50 and 24 design points, respectively [3]. The ANN is a feed-forward network and the optimal number of hidden nodes is nine, which has been automatically determined within a specified range by the ANN tool. Furthermore it has been found that one hidden layer was sufficient and that within the relevant range of design parameters the approximation is acceptable with an error of about $1 \%$.

Once the ANN is available, the multi objective optimisations can be performed with the different optimisation algorithms. The GA is run with a population of 100 and for 30 generations. The initial population is taken randomly from the 342 input vectors and will contain about 22 rank-one points in the output space. For both the minimax method and the equality-constraint method those inputs of the training set are chosen which have rank-one outputs. There are 55 of those input vectors.

For each individual method, after the optimisation is run, the rank-one points are selected and taken as approximation of the Pareto front. The results are depicted in Fig. 6. In Table 1 the approximate number of function calls, the total number of 
points resulting from the optimisation, and the number of points in the non-dominated sets are shown.
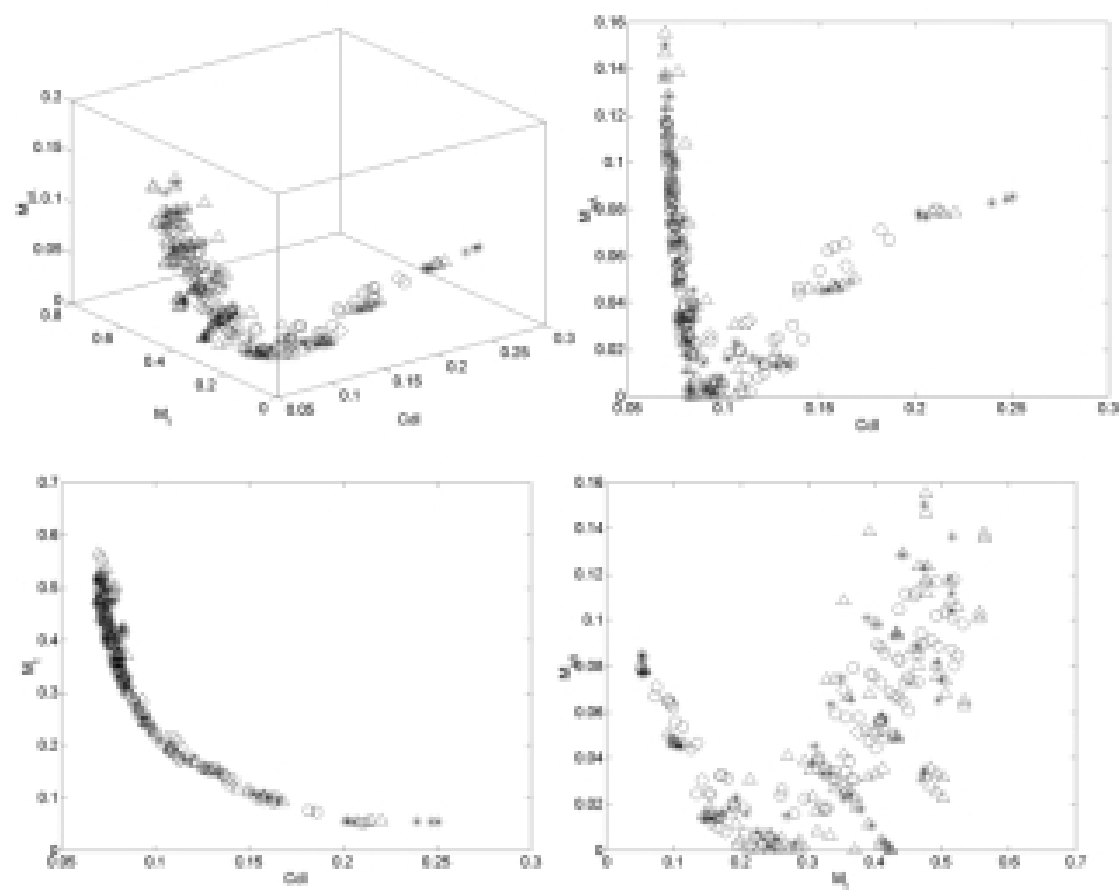

Fig. 6. The results of the different optimisation methods are depicted here. The $C_{d l}, M_{t}$ and $M_{p A}$ obtained by the GA (o), minimax method (*) and the equality-constraint method $(\Delta)$ are depicted in 3D and in 2D projections.

Table 1. Some numbers about the performance of each individual method

\begin{tabular}{|l|l|l|l|}
\hline Method & GA & minimax & equality-constraint \\
\hline Total nr of points in final result & 100 & 55 & 165 \\
\hline Nr of non-dominated points & 100 & 54 & 113 \\
\hline Number of function calls (about) & 4900 & 2000 & 6400 \\
\hline
\end{tabular}

The rank-one points for each method can than be compared with the rank-one points of the other methods. Then sub-optimal solutions (i.e. not rank-one) can then be found. In Table 2 the results are summarised. One sees that every method yields some sub-optimal points. 
Table 2. The number of non-dominated and dominated points in set \#1 when comparing the set with set \#2. The numbers left of the slash are the non-dominated points, while those right of the slash are the dominated points.

\begin{tabular}{|l|l|l|l|}
\cline { 2 - 4 } \multicolumn{1}{c|}{} & \multicolumn{3}{l|}{ set \#2 } \\
\hline set \#1 & GA & minimax & GM \\
\hline GA & - & $96 / 4$ & $93 / 7$ \\
\hline Minimax & $51 / 3$ & - & $51 / 3$ \\
\hline GM & $108 / 5$ & $100 / 13$ & - \\
\hline
\end{tabular}

\section{Discussion and conclusions}

In this paper an ICT environment for multi objective optimisation and multidisciplinary design has been presented. Special attention has been paid to the functionality for multi objective optimisation and approximate modeling. Several methods for multi objective optimisation, based on GA and GM, and a method for approximate modeling based on ANN, were implemented in this environment. Furthermore, several tools for specific aeronautic design analyses have been adopted from the MDO environment (the $\mathrm{CDE}$ ) that is developed in the MOB project.

The ICT environment has been applied to a predesign case of the BWB aircraft configuration from the MOB project. In the multi objective optimisation analysis an ANN approximation of the CFD calculations has been made, and an initial comparative study of the use of the GA and two of the GM based methods for MOO has been performed. The results indicate that the different multi objective optimisation methods provide comparable results at comparable computational cost. However, the robustness and global search capabilities of the GA compete with the higher efficiency of the GM due to the smaller size of the required sets of design points. Therefore these methods have complementary functionality.

\section{Acknowledgements}

The support of Dr. M. Laban and Dr. J.C. Kok in setting up the CFD simulations of the BWB with NLR's Enflow system is gratefully acknowledged. This study has been partially supported by the EU project MOB, contract number G4RD-CT1999-0172.

\section{References}

1. Allwright, S.: Multi-discipline Optimisation in Preliminary Design of Commercial Transport Aircraft. In: Proc. ECCOMAS'96 Conference, Paris (1996)

2. Baalbergen, E.H., van der Ven, H.: SPINEware - A Framework for User-Oriented and Tailorable Metacomputers, Future Generation Computer Systems 15 (1999) 549-558

3. Bishop. Chr. M: Neural Networks for Pattern Recognition. Clarendon Press, Oxford (1997)

4. Fonseca, C.M., Fleming, P.J.: Genetic Algorithms for Multi-Objective Optimization: Formulation, Discussion and Generalization. In: Genetic Algorithms: Proc. of the Fifth Int. Conf., San Mateo, CA, USA (1993) 416-423

5. Fonseca, C.M., Fleming, P.J.: An Overview of Evolutionary Algorithms in MultiObjective Optimization. Evolutionary Computing 3 (1995) 1-16 
6. The MathWorks: Matlab Optimization Toolbox. http://www.mathworks.com/products/ optimization.

7. The MathWorks: Matlab Neural Network Toolbox. http://www.mathworks.com/products/ neural.

8. Pareto, V.: Manuale di Economia Politica. Sociata Editrice Libraria, Milan, Italy (1906).

9. Sawaraki, Y., and Nakayama, H.: The Theory of Multiobjective Optimization. Academic Press, Inc, (1985)

10. Schultheiss, B.C., Baalbergen, E.H.: Utilizing Supercomputer Power from your Desktop. HPCN 2001 Conference, Amsterdam (2001)

11. Tan, K.C.,: MOEA - Multi-objective Evolutionary Algorithm. http://vlab.ee.nus.edu.sg/ $\sim \mathrm{kctan}$.

12. Vogels M.E.S., Arendsen, P., Krol, R.J., Laban M., and Pruis, G.W.: From a Monodisciplinary to a Multidisciplinary Approach in Aerospace: As Seen from Information and Communication Technology Perspective. In: ICAS 98, Melbourne, Australia (1998)

13. "MOB: A Computational Design Engine Incorporating Multi-disciplinary Design and Optimisation for Blended-Wing-Body Configuration". EC $5^{\text {th }}$ Framework Programme, Contract nr. GRD1-1999-11162 (2000).

14. Shyy W., Nilay P., Vaidyanathan R., Tucker K.: Global design optimization for aerodynamics and rocket proulsion components. Progress in Aerospace Sciences 37 (2001) 59-118. 